\title{
Detection of non-obstructive coronary artery disease: Is post-stress diastolic dysfunction assessed by myocardial perfusion imaging a useful tool?
}

\author{
Bernard Songy, $M^{a}$ \\ ${ }^{a}$ Nuclear Cardiology, Centre Cardiologique du Nord (CCN), Paris, France
}

Received Mar 21, 2016; accepted Mar 22, 2016

doi: $10.1007 / \mathrm{s} 12350-016-0486-3$

\section{See related article, pp. 1542-1550}

In the paper entitled «Myocardial ischemia in the absence of obstructive coronary lesion: the role of poststress diastolic dysfunction in detecting early coronary atherosclerosis $\gg$, Gimelli et al evaluated the interactions between non-obstructive coronary atherosclerosis on invasive coronary angiography (ICA) and myocardial perfusion imaging (MPI) with a cadmium-zinc-telluride (CZT) camera. ${ }^{1}$ They calculated the summed stress score (SSS) for perfusion, left ventricular ejection fraction (LVEF) for global systolic function, and peak filling rate (PFR) for global diastolic function. This preliminary paper concludes that in patients with chest pain, despite the absence of anatomically obstructive coronary artery disease (CAD), the alteration of post-stress left ventricular diastolic function associates with the presence of non-obstructive atherosclerosis on ICA. In these subjects, the degree of post-stress left ventricular diastolic impairment correlates with myocardial perfusion abnormalities on MPI, despite the absence of a relevant reduction in post-stress LVEF. The authors suggest that a combined evaluation of myocardial perfusion and diastolic function may better detect the presence of early stages of CAD. This paper and its conclusions lead us to ask three key questions:

\footnotetext{
Reprint requests: Bernard Songy, MD, Nuclear Cardiology, Centre Cardiologique du Nord (CCN), Paris, France; b.songy@ ccncardio.com J Nucl Cardiol 2017;24:1551-4.

1071-3581/\$34.00

Copyright (C) 2016 American Society of Nuclear Cardiology.
}

- Is the detection of non-obstructive CAD helpful?

- Is post-stress diastolic dysfunction an adequate marker?

- Is MPI able to detect post-stress diastolic dysfunction and, thus, non-obstructive CAD?

\section{DETECTION OF NON-OBSTRUCTIVE CAD}

CAD remains one of the major causes of mortality. Classically, it has been acknowledged that stress coronary flow decreases significantly only when narrowing exceeds $70 \%{ }^{2}$ Stenoses with less than $50 \%$ narrowing were supposed not to affect coronary flow reserve and stress myocardial perfusion abnormalities were therefore generally considered to be false-positive findings. More recently, fractional flow reserve (FFR) obtained by pressure wire with ICA has shown a poor correlation between artery narrowing and loss of pressure. Thus, FFR is abnormal $(<0.80)$ in $35 \%$ of patients with stenosis between $50 \%$ and $70 \%$ narrowing. ${ }^{3}$ FFR may also be abnormal in patients with stenosis having less than $50 \%$ narrowing. ${ }^{4,5}$ Vasospastic angina may occur in patients with non-obstructive atherosclerosis. While it usually happens at rest and only occasionally with exertion in patients with obstructive atherosclerosis, exercise-induced spastic angina is more often observed in patients with non-obstructive atherosclerosis. ${ }^{6}$ Moreover, acute coronary syndromes (ACS) are often due to non-obstructive and non-calcified atherosclerotic plaques. Indeed, obstructive stenoses are more often fibrotic and poorly cellular, with thick, fibrous caps. In contrast, culprit lesions in ACS show the rupture of a thin fibrous cap, with exposure towards the lumen of large, soft, prothrombotic, necrotic core material that can trigger occlusive or non-occlusive thrombosis. ${ }^{7}$ Distal embolization with non-obstructive stenosis has been raised in women. ${ }^{8}$ Coronary reactivity dysfunction 
seems to be common in non-obstructive CAD and may also be considered. ${ }^{9}$ Endothelial dysfunction results in a lack of response to factors of arterial dilatation, especially nitric oxide (NO). Microvascular dysfunction (endothelial independent) is related to impaired smooth muscle relaxation and results in decreased coronary flow reserve. Both are associated with a higher rate of cardiac events. $^{10-12}$ In hypertensive post-menopausal women, the restoration of endothelial function was associated with improved outcome. ${ }^{13}$ Statins and ACE inhibitors improve endothelial dysfunction and may benefit patients with non-obstructive CAD. Coronary reactivity dysfunction induces a reduced coronary flow reserve, which may cause later interstitial fibrosis, resulting in impaired diastolic function. ${ }^{14}$ For all of these reasons, the identification of non-obstructive atheroma should be considered, even if the clinical implication remains unclear. The most common method for diagnosing subclinical $\mathrm{CAD}$ is measurement of a coronary artery calcium score (CACS). ${ }^{15}$ Until now, MPI was used to assess the functional consequences of obstructive CAD and showed no perfusion or systolic function abnormalities in nonobstructive CAD. Patients without known CAD undergoing stress MPI could undergo a same-setting coronary calcification study. ${ }^{16}$ However, CACS underestimates the presence of an atherosclerotic plaque burden, ignoring soft, non-calcified plaques. But coronary artery CT angiography may show non-calcified, non-obstructive coronary stenosis, even if it fails to quantify it accurately.

\section{POST-STRESS DIASTOLIC DYSFUNCTION}

Diastolic dysfunction (DD) results in ineffective left atrial emptying and left ventricular filling, reduces the ability to augment cardiac output with exercise, increases pulmonary pressure, and results in symptoms and fluid retention. Diastolic relaxation and filling appear to be altered by ischemia, which lead to asynchronous myocardial relaxation and, thus, global DD. The pathophysiology of systolic and diastolic dysfunction is quite different: while systolic dysfunction seems to be a disease of calcium handling, DD seems to be an increased myofilament sensitivity to calcium. ${ }^{17}$ Diastolic function seems more susceptible to ischemia than systolic function. Therefore, DD precedes the onset of systolic dysfunction and takes longer to resolve. ${ }^{18}$ In ACS, DD assessed by MRI remains 24 hours after reflow in regions underperfused during coronary occlusion and in the absence of infarct, whereas regional systolic contractility has recovered. DD is more marked when microvascular obstruction is present at the tissue level after reopening of the coronary artery. ${ }^{19} \mathrm{DD}$ is also impaired in stable obstructive CAD. Increased left ventricular end-diastolic pressure (LVEDP) measured by left cardiac catheterization is correlated with the severity and the extent of obstructive CAD, evaluated by invasive coronary angiography (ICA). ${ }^{20}$ Non-obstructive CAD can also result in DD: non-obstructive CAD assessed by computed tomography coronary angiography (CTCA) is associated with increased left ventricular pressure (LVFP) measured by echography; ${ }^{21}$ in patients without ischemia, an abnormal coronary calcium score in the absence of ischemia is associated with echographic parameters of DD. ${ }^{22}$ In women with signs and symptoms of ischemia in the absence of obstructive CAD, DD was observed with cardiac MRI. ${ }^{8}$ Thus, diastolic function is impaired both in obstructive CAD, correlated with the extent and severity of obstructive disease, and in non-obstructive CAD. However, DD is not specific to obstructive or non-obstructive CAD. Preclinical diastolic dysfunction (PDD), i.e., DD with normal systolic function and no symptoms of heart failure, is prevalent in the general adult population (approximately $20 \%$ to $30 \%$, increasing with age, hypertension, and diabetes), but remains poorly understood. ${ }^{17}$ Available data suggest that patients with PDD are diverse. DD is known to be associated with left ventricular hypertrophy ( $\mathrm{LVH})$, but recent investigations suggest that DD precedes the development of $\mathrm{LVH}$. Moreover, regression of LVH with antihypertensive treatment results in little change in DD parameters. ${ }^{17}$ Despite the fact that DD is considered the first stage of diastolic disease with natural progression to heart failure with preserved ejection fraction (HFpEF), ${ }^{8,14,17}$ inconsistent features may be pointed out: PDD is associated with cardiovascular risk factors, such as hyperlipidemia, diabetes, and hypertension, but also with peripheral vascular disease; conversely, non-cardiac risk factors, such as renal impairment, pulmonary airflow limitations, or anemia, are involved in the progression of DD to HFpEF; therapeutic strategies focusing on cardiovascular risk factors and comorbidities have not been shown to be effective in improving outcome in HFpEF. ${ }^{17}$ One might therefore wonder whether DD with ischemia in the absence of obstructive CAD is the early stage of a diastolic function disease which will progress to HFpEF or whether DD is an independent marker associated with cardiovascular risk factors or peripheral arterial disease.

\section{POST-STRESS DIASTOLIC DYSFUNCTION ASSESSED BY MPI}

The concept of stress DD has been introduced with stress echography. MPI has the advantage of acquiring images 10 to 15 minutes after exercise, avoiding an elevated heart rate, which may perturb the evaluation of left ventricular dynamics, thus allowing persistent DD 
evaluation and lower cardiac rate to be combined. The MPI protocol described for the assessment of diastolic function used a CZT camera (Discovery NM 530c, Ge Healthcare, Haifa, Israel), of which the myocardial count efficiency is sixfold that of a conventional camera. ${ }^{23} 99 \mathrm{~m}$ Tc-tetrofosmin was injected at stress (185-222 MBq, i.e., 5-6 mCi) then at rest (370$444 \mathrm{MBq}$, i.e., $10-12 \mathrm{mCi}$ ) with a list mode acquisition of 7 minutes and an iterative reconstruction (50 iterations), without scatter or attenuation correction. Left ventricular function analysis was performed from 16frame reformatted images using commercially available software (Corridor 4DM, Invia, Ann Arbor, Mi, USA). ${ }^{24}$ Besides left ventricular end-diastolic volume (EDV), end-systolic volume (ESV), and ejection fraction (EF), the left ventricular volume curve was fitted with a fourth-order harmonic function in order to derive the PFR, expressed in EDV/s as an indicator of left ventricular diastolic function. Measurements were obtained at stress and rest and measures of PFR were corrected for the R-R interval. ${ }^{25}$ Characteristics of the studied population (mean age of 69 years, male gender in $65 \%$ of cases, hypertension in $57 \%$ of cases) avoided major bias, given the prevalence and the polymorphism of DD reported above. At rest, PFR was equal to $2.7 \pm 0.8$ in normal patients, $2.5 \pm 0.7$ in non-obstructive CAD, $2.5 \pm 0.7$ in one-vessel obstructive $C A D$, and $2.3 \pm 0.6$ in three-vessel disease. At stress, differences were more marked, with PFR equal to $2.9 \pm 0.8$ in normal patients, $2.5 \pm 0.9$ in non-obstructive CAD, $2.5 \pm 0.7$ in one-vessel obstructive $\mathrm{CAD}$, and $2.1 \pm 0.67$ in three-vessel disease. ${ }^{26}$ These results confirm that post-stress evaluation of diastolic function is more discriminative than rest evaluation. They show that non-obstructive $\mathrm{CAD}$ has a profile that differs from that of a normal coronary artery, but is similar to that of single-vessel obstructive CAD. Additionally, the accuracy of these results illustrates the potential of MPI to assess stress DD. However, the large standard deviations and overlap between normal, non-obstructive $\mathrm{CAD}$, and obstructive CAD limit the information that could be utilized for a given patient. With a stress PFR cutoff of 2.0 , only $28 \%$ of non-obstructive CAD cases are detected and $13 \%$ of normal patients are over-diagnosed. However, it is strongly suggested that stress DD could be an additional tool for more complete characterization of CAD. Therefore, MPI might give, at the same time, incremental information about perfusion and systolic and diastolic function.

\section{CLINICAL IMPLICATIONS}

Due to the preliminary character and limitations of the aforementioned study, particularly the overlap between patients with non-obstructive CAD and those without CAD, further studies are needed. At this point in time, stress DD is an interesting pathophysiological concept, but does not provide diagnostic support. Up to now, clinical implications remain unclear; however, several options may be suggested: The assessment of diastolic function with MPI in the routine clinical setting would provide additional data when obstructive CAD is present and could suggest the existence of non-obstructive CAD when no ischemia or mild ischemia is detected. It would also be worthwhile to study the added value of combined CACS. On the other hand, the association of MPI and CTA would give additional information about coronary anatomy, myocardial perfusion, and systolic and diastolic function. The challenge could be to identify high-risk subnormal patients. For example, we could ask if the outcome would be the same in patients with non-calcified and non-obstructive stenosis, with or without stress DD in MPI.

\section{References}

1. Gimelli A, Liga R, Pasanisi EM, Casagranda M, Marzullo P. Myocardial ischemia in the absence of obstructive coronary lesion: The role of post-stress diastolic dysfunction in detecting early coronary atherosclerosis. J Nucl Cardiol 2016;. doi: 10.1007/s12350-016-0456-9.

2. Gould KL, Lipscomb K. Effects of coronary stenoses on coronary flow reserve and resistance. Am J Cardiol 1974;34:48-55.

3. Tonino PA, Fearon WF, De Bruyne B, Oldroyd KG, Leesar MA, Ver Lee PN, et al. Angiographic versus functional severity of coronary artery stenoses in the FAME study fractional flow reserve versus angiography in multivessel evaluation. J Am Coll Cardiol 2010;55:2816-21.

4. Meijboom WB, Van Mieghem CA, van Pelt N, Weustink A, Pugliese F, Mollet NR, et al. Comprehensive assessment of coronary artery stenoses: Computed tomography coronary angiography versus conventional coronary angiography and correlation with fractional flow reserve in patients with stable angina. J Am Coll Cardiol 2008;52:636-43.

5. Gould KL. Does coronary flow trump coronary anatomy? JACC Cardiovasc Imaging 2009;2:1009-23.

6. Yilmaz A, Hill S, Schäufele T, Vöhringer M, Geissler A, Sechtem U. Exercise-induced spastic coronary artery occlusion at the site of a moderate stenosis: Neither Prinzmetal's angina nor cardiac syndrome X but “Prinzmetal X'. Circulation 2010;122:e570-4.

7. Montalescot G, Sechtem U, Achenbach S, Andreotti F, Arden C, Budaj A, et al. 2013 ESC guidelines on the management of stable coronary artery disease: The task force on the management of stable coronary artery disease of the European Society of Cardiology. Eur Heart J 2013;34:2949-3003.

8. Nelson MD, Szczepaniak LS, Wei J, Haftabaradaren A, Bharadwaj M, Sharif B, et al. Diastolic dysfunction in women with signs and symptoms of ischemia in the absence of obstructive coronary artery disease: a hypothesis-generating study. Circ Cardiovasc Imaging 2014;7:510-6.

9. Shaw J, Anderson T. Coronary endothelial dysfunction in nonobstructive coronary artery disease: Risk, pathogenesis, diagnosis and therapy. Vasc Med 2016;21:146-55. 
10. Reis SE, Holubkov R, ConradSmith AJ, Kelsey SF, Sharaf BL, Reichek N, et al. Coronary microvascular dysfunction is highly prevalent in women with chest pain in the absence of coronary artery disease: Results from the NHLBI WISE study. Am Heart J 2001;141:735-41.

11. Halcox J, Schenke WH, Zalos G, Mincemoyer R, Prasad A, Waclawiw MA, et al. Prognostic value of coronary vascular endothelial dysfunction. Circulation 2002;106:653-8.

12. Ong P, Athanasiadis A, Borgulya G, Mahrholdt H, Kaski JC, Sechtem U. High prevalence of a pathological response to acetylcholine testing in patients with stable angina pectoris and unobstructed coronary arteries. The ACOVA Study (abnormal coronary vasomotion in patients with stable angina and unobstructed coronary arteries). J Am Coll Cardiol 2012;59:655-66.

13. Modena MG, Bonetti L, Coppi F, Bursi F, Rossi R. Prognostic role of reversible endothelial dysfunction in hypertensive postmenopausal women. J Am Coll Cardiol 2002;40:505-10.

14. Shaw LJ, Bugiardini R, Merz CN. Women and ischemic heart disease: Evolving knowledge. J Am Coll Cardiol 2009;54:156175 .

15. Bateman TM, Heller GV, McGhie AI, Friedman JD, Case JA, Bryngelson JR, et al. Diagnostic accuracy of rest/stress ECG-gated Rb-82 myocardial perfusion PET: Comparison with ECG-gated Tc-99m sestamibi SPECT. J Nucl Cardiol 2006;13:24-33.

16. Bybee KA, Lee J, Markiewicz R, Longmore R, McGhie AI, O'Keefe JH, et al. Diagnostic and clinical benefit of combined coronary calcium and perfusion assessment in patients undergoing PET/CT myocardial perfusion stress imaging. J Nucl Cardiol 2010;17:188-96.

17. Wan SH, Vogel MW, Chen HH. Pre-clinical diastolic dysfunction. J Am Coll Cardiol 2014;63:407-16.

18. Paul AK, Kusuoka H, Hasegawa S, Yonezawa T, Makikawa M, Nishimura T. Prolonged diastolic dysfunction following exercise induced ischaemia: A gated myocardial perfusion SPECT study. Nucl Med Commun 2002;23:1129-36.
19. Garot J. The study of diastole by tagged MRI: are we nearly there yet? Eur Heart J 2004;25:1376-7.

20. Du LJ, Dong PS, Jia JJ, Fan XM, Yang XM, Wang SX, et al. Association between left ventricular end-diastolic pressure and coronary artery disease as well as its extent and severity. Int J Clin Exp Med 2015;8:18673-80.

21. Lin FY, Zemedkun M, Dunning A, Gomez M, Labounty TM, Asim M, et al. Extent and severity of coronary artery disease by coronary CT angiography is associated with elevated left ventricular diastolic pressures and worsening diastolic function. J Cardiovasc Comput Tomogr 2013;7:289-96.

22. Maragiannis D, Schutt RC, Gramze NL, Chaikriangkrai K, McGregor K, Chin K, et al. Association of left ventricular diastolic dysfunction with subclinical coronary atherosclerotic disease burden using coronary artery calcium scoring. J Atheroscler Thromb 2015;22:1278-86.

23. Imbert L, Poussier S, Franken PR, Songy B, Verger A, Morel O, et al. Compared performance of high-sensitivity cameras dedicated to myocardial perfusion SPECT: A comprehensive analysis of phantom and human images. J Nucl Med 2012;53:1897-903.

24. Ficaro EP, Lee BC, Kritzman JN, Corbett JR. Corridor4DM: The Michigan method for quantitative nuclear cardiology. J Nucl Cardiol 2007; 14:455-65.

25. Gimelli A, Liga R, Pasanisi EM, Giorgetti A, Marras G, Favilli B, et al. Evaluation of left ventricular diastolic function with a dedicated cadmium-zinc-telluride cardiac camera: Comparison with Doppler echocardiography. Eur Heart J Cardiovasc Imaging 2014;15:972-9.

26. Gimelli A, Liga R, Bottai M, Pasanisi EM, Giorgetti A, Fucci S, et al. Diastolic dysfunction assessed by ultra-fast cadmium-zinctelluride cardiac imaging: Impact on the evaluation of ischaemia. Eur Heart J Cardiovasc Imaging 2015;16:68-73. 